\title{
Novel scheme for code preserving regenerative NRZ-DPSK wavelength and format conversion
}

\author{
M. Presi, N. Calabretta, G. Contestabile and E. Ciaramella \\ Scuola Superiore Sant'Anna, Via G. Moruzzi 1, I-56124 Pisa, Italy, email: marco.presi@sssup.it
}

\begin{abstract}
We propose a novel code-preserving NRZ-DPSK wavelength and format converter. It is based on optical filtering and a bi-stable device. It can be applied both to continuos and packet/burst traffic. Regenerative capability is also demonstrated.

(c) 2008 Optical Society of America

OCIS codes: (230.7405) Wavelength conversion devices; (200.6015) Signal regeneration
\end{abstract}

\section{Introduction}

Interned traffic boosted the study of sub-systems that, interconnecting transparently the layered networks, enable the realization of high capacity and flexible optical networks. Indeed, while OOK signals will be more likely used in metro-access network, DPSK signals, owing its higher resilience to propagation impairments [1], will be probably used in long-haul-metro networks. Beside the several all-optical circuits for packets manipulation, wavelength conversion (WC) and format conversion (FC) are key functions for transparent packet routing and interconnecting different layered optical networks. Critical issues in developing practical DPSK-WC and FC for optical networks are the differential coding preservation, due to the unpredictability of the number of crossed nodes by the packet, and the regeneration capability that allows cascadability of the circuits. DPSK-WC based on four-wave-mixing preserves not only the encoding, but also the modulation format [2]. However, limited cascadability, high operating power, and polarization dependency strongly limit this technique. Alternative DPSK-WC solutions are based on non-linear processes in SOAMZI structures [3]. They first demodulated the original DPSK signal obtaining an OOK signal (and its inverted copy), and then used those as push-and-pull signals in an SOA-MZI structure, acting as an optical phase modulator (OPM), for OOK to BPSK converting the signal at different wavelength. In [4] the OPM was implemented by exploiting the Kerr effect in a non-linear fiber. An optical DPSK encoder should be used to retrieve the original information, but it has never been demonstrated. Here, we present a novel technique that allows the first implementation of a coding-preserving DPSK-WC with no optical DPSK encoder. The basic idea is to use the information contained in the phase-transitions of the original DPSK signal to drive an optical bistable circuit (OBC). The obtained OOK signal contains the same pattern (in intensity) of the phase pattern of the original DPSK signal, and can directly drive an OPM to complete the WC processing.
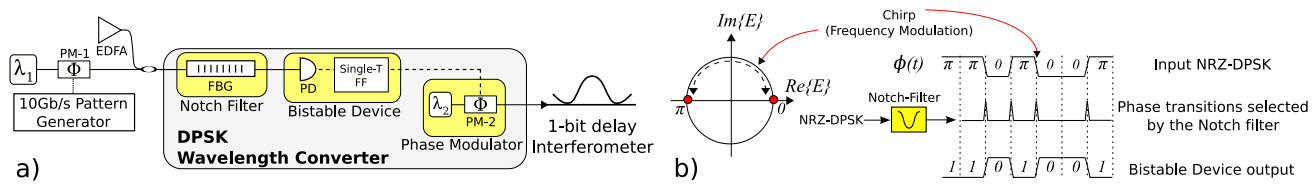

Fig. 1. a) Operating principle of the DPSK-WC. b) Experimental set-up.

\section{Experiments}

The schematic of the proposed DPSK-WC is shown in fig. 1-b. It consists of a notch filter, a bistable device and a phase modulator. The operating principle is illustrated in fig. 1-a, where, for better clarity, we report also the well-known NRZ-DPSK constellation diagram. In NRZ-DPSK format, the phase-modulation is achieved by moving the optical field $\mathrm{E}(\mathrm{t})$ over the magnitudine-constant path shown in fig. 1-a, thus realizing a constant envelope signal. The phase is changed continuously between 0 and $\pi$, thus every transition brings high-frequency components. These can be isoleted from the DPSK signal by using an optical notch filter centered at the signal carrier wavelength. The notch-filter output is composed by an optical pulse at every phase-transition in the incoming signal, as sketched in fig. 1-a (see also the measurements in fig. 2-b). Such pulses should drive a OBC (such as an optical set-reset flip-flop), thus creating an intensity NRZ binary pattern (see fig. 2-c) that matches exactly (either in direct or inverse logic) the phase pattern of the incoming signal (see fig. 2-a). By using an OPM, the intensity pattern at the output of the bistable element can be 
converted to a phase pattern at one or multiple different wavelengths. The converted signal preserves the coding of the original DPSK signal. The esperimental setup is reported in fig. 1-b. A CW source at $1549.8 \mathrm{~nm}$ was modulated by a $\mathrm{LiNbO}_{3}$ phase-modulator, producing no spurious intensity modulation. An user pattern bit sequence (see fig. 2-a) was used to easily show the effectively coding preserving along different points in the set-up. The data bit-rate was $10 \mathrm{~Gb} / \mathrm{s}$. The DPSK signal was amplified and fed into the notch filter, which consisted of an athermal FBG. The FBG had a Gaussian profile with a 3-dB bandwidth of $6 \mathrm{GHz}$ and an almost flat phase response and no spurious dispersion. The ouput of the FBG is reported in fig. 2-b, which clearly show the optical pulses occurring at every phase-transition of the DPSK signal. The optical pulses had a pulsewidth of $45 \mathrm{ps}$ and an extinction ratio of around $12 \mathrm{~dB}$. Those pulses were fed into a OBC. In our demonstration, the lack of an optical flip-flop forced us to use an electrical flip-flop (FF). The optical pulses were then converted to electrical pulses by a fast photodiode before to be fed into a toggle FF with $20 \mathrm{GHz}$ maximum operating rate. The electrical signal at the FF output is reported in fig. 2-c. The figure clearly
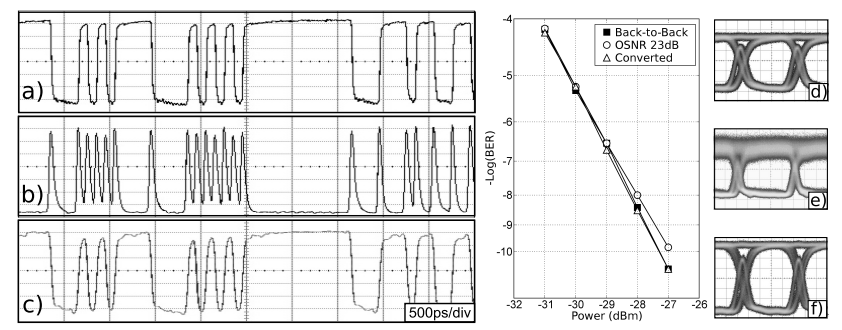

Fig. 2. Right: a) Input electrical DPSK signal. b) FBG optical output pulses. c) Output of the electrical flip-flop. Left: Bit error rate measurements of the d) input, e) degraded, and g) converted DPSK signals.

shows the match between the original electrical coded DPSK signal and the signal after the FF. This confirms that our proposed WC preserves the differential encoding. To complete the WC operation, the electrical signal provided by the FF was used to drive a phase modulator, which modulated the phase of one (or multiple) CW source at different wavelength. Note that in case an optical FF would have been available, the produced FF output would be an optical NRZ-OOK that may all-optically used to drive an OPM similar as that employed in [4]. The performance of the DPSK-WC was also quantified by the BER measurements, reported in fig. 2. To investigate the regenerative capability of this WC, we degraded an input DPSK signal by adding in-band ASE noise produced by an EDFA. The OSNR was degraded to $23 \mathrm{~dB}$. Due to the added noise, the degraded signal presented a penalty at $10^{-9}$. After the wavelength conversion, no appreciable penalty was measured. Indeed, the figure clearly shows that the added noise is cleaned after the conversion stage (see eyes in the of fig. 2). This is due to the decisional and limiting characteristic of the FF: once the FF was switched its state, any bit noise fluctuations were completely eliminated. The removal of the penalty and the regenerated OSNR due to wavelength conversion confirm a regenerative capability and the potential cascadability of the proposed circuit.

We demonstrated a novel technique that allows the implementation of a phase preserving DPSK-WC without an optical DPSK encoder. The device can be of high interest in wavelength conversion for DPSK signal in optical networks where optical packets cross an unpreadictable number of nodes. Error-free operation of the wavelength converter was experimentally demostrated, even for input signals with a degraded OSNR of $23 \mathrm{~dB}$. This indicates the potential cascadabilidy of the circuit. Finally, we point out that althought the proof-of-principle was demonstrated also by means of optoelectronics components (namely the electrical FF and the phase modulator), those could be replaced by all-optical components, which were all already demonstrated (an optical set-reset flip-flop [5] and an optical phase modulator [4], respectively).

\section{References}

1. A.H. Gnauck and P. J. Winzer, "Optical phase-shift keyed transmission,” J. Lightwave Technol., vol. 23, no. 1, pp. 115-130, 2005.

2. P.A. Andersen, T. Tokle, Y. Geng, C. Peucheret, and P. Jeppsen, "Wavelength conversion of a 40-Gb/s RZ-DPSK signal using four-wave mixing in a dispersion-flattened highly nonlinear photonic crystal fiber," IEEE Photon. Technol. Lett., vol. 17, no. 9, pp. 1908-1910, 2005.

3. I. Kang, C. Dorrer, L. Zhang, M. Rasras, L. Buhl, A. Bhardwaj, S. Cabot, M. Dinu, X. Liu, M. Cappuzzo, L. Gomez, A. Wong-Foy, Y.F. Chen, S. Patel, D.T. Neilson, J. Jacques, and C.R Giles, "Regenerative all-optical wavelength conversion of $40 \mathrm{~Gb} / \mathrm{s}$ DPSK signals using a SOA MZI," in ECOC 2005, vol. 6, 2005, pp. 29-30.

4. C. Schmidt-Langhorst, R. Ludwig, M. Galili, B. Huettl, F. Futami, S. Watanabe, and C. Schubert, "160 Gbit/s all-optical OOK to BPSK in-line format conversion," in ECOC 2006, Cannes, France, no. Th4.3.5.

5. M. T. Hill, H. J. S. Dorren, T. de Vries, X. J. M. Leijtens, J. Hendrik den Besten, B. Smalbrugge, Y.S. Oei, H. Binsma, G.D.Khoe, and M. K. Smit, "A fast low-power optical memory based on coupled micro-ring lasers," Nature, no. 432, pp. 206-209, 2004. 